\title{
BMJ Open Randomised controlled trial of a financial incentive for increasing the number of daily walking steps: study protocol
}

\author{
Yasutake Tomata, ${ }^{\oplus 1}$ Fumiya Tanji, ${ }^{1}$ Dieta Nurrika, ${ }^{1}$ Yingxu Liu, ${ }^{1}$ Saho Abe, ${ }^{1}$ \\ Koichi Matsumoto, ${ }^{1}$ Shu Zhang, ${ }^{1}$ Yumika Kotaki, ${ }^{1}$ Sanae Matsuyama, ${ }^{1}$ Yukai Lu, ${ }^{1}$ \\ Yumi Sugawara, ${ }^{1}$ Shino Bando, ${ }^{1}$ Teiichiro Yamazaki, ${ }^{1}$ Tatsui Otsuka, ${ }^{1}$ \\ Toshimasa Sone, ${ }^{1,2}$ Ichiro Tsuji ${ }^{1}$
}

To cite: Tomata Y, Tanji F, Nurrika D, et al. Randomised controlled trial of a financial incentive for increasing the number of daily walking steps: study protocol. BMJ Open 2019;9:e026086. doi:10.1136/ bmjopen-2018-026086

- Prepublication history for this paper is available online. To view these files, please visit the journal online (http://dx.doi org/10.1136/bmjopen-2018026086).

Received 16 August 2018 Revised 20 May 2019 Accepted 30 May 2019

Check for updates

(c) Author(s) (or their employer(s)) 2019. Re-use permitted under CC BY-NC. No commercial re-use. See rights and permissions. Published by BMJ.

${ }^{1}$ Division of Epidemiology, Department of Health Informatics and PublicHealth, Tohoku University Graduate School of Medicine, Sendai, Japan

2Department of Rehabilitation, Faculty of Health Science, Tohoku Fukushi University, Sendai, Japan

Correspondence to Dr Yasutake Tomata; y-tomata@med.tohoku.ac.jp

\section{ABSTRACT}

Introduction Physical activity is one of the major modifiable factors for promotion of public health. Although it has been reported that financial incentives would be effective for promoting health behaviours such as smoking cessation or attendance for cancer screening, few randomised controlled trials (RCTs) have examined the effect of financial incentives for increasing the number of daily steps among individuals in a community setting. The aim of this study is to investigate the effects of financial incentives for increasing the number of daily steps among community-dwelling adults in Japan.

Methods and analysis This study will be a two-arm, parallel-group RCT. We will recruit community-dwelling adults who are physically inactive in a suburban area (Nakayama) of Sendai city, Japan, using leaflets and posters. Participants that meet the inclusion criteria will be randomly allocated to an intervention group or a waitlist control group. The intervention group will be offered a financial incentive (a chance to get shopping points) if participants increase their daily steps from their baseline. The primary outcome will be the average increase in the number of daily steps (at 4-6 weeks and 7-9 weeks) relative to the average number of daily steps at the baseline (1-3 weeks). For the sample size calculation, we assumed that the difference of primary outcome would be 1302 steps.

Ethics and dissemination This study has been ethically approved by the research ethics committee of Tohoku University Graduate School of Medicine, Japan (No. 20181-171). The results will be submitted and published in a peer-reviewed scientific journal.

Trial registration number UMIN000033276; Pre-results.

\section{INTRODUCTION}

Physical activity is a major modifiable factor that has benefits in terms of physical and mental health. ${ }^{1}$ Therefore, public health strategies to increase physical activity are implemented worldwide. ${ }^{2}$ In the Japanese National Health Promotion Movement ('Health Japan

\section{Strengths and limitations of this study}

- This trial will examine the effectiveness of a financial incentive for increasing the number of daily walking steps.

- The present study would be first Asian randomised controlled trials of financial incentives intervention.

- Limitations include the fact that the intervention will be only one type of financial incentive.

- Only short-term effects during 9 weeks will be evaluated.

21'), a higher number of daily walking steps is a target for physical activity. ${ }^{34}$

Recently, to encourage individuals who are not concerned about health-related behaviour to increase the number of steps they walk daily, it has been suggested that offering them financial incentives might be an effective approach. The Ministry of Health, Labor and Welfare in Japan has provided a guideline for promotion of public health using financial incentives. ${ }^{5}$ One such incentive is the introduction of a 'health point system' in which local governments provide 'shopping points' that can be redeemed in local stores when an individual achieves a health-related behaviour goal such as an increase in the number of daily walking steps.

A systematic review (meta-analysis) has suggested that financial incentives would be effective for promotion of health behaviours such as smoking cessation or attendance for vaccination or cancer screening. ${ }^{6}$ Although a few randomised controlled trials (RCTs) (financial incentives vs no intervention) have examined the effect of financial incentives for increasing the amount of daily steps by individuals in a community setting, the results were not consistent. ${ }^{78}$ One previous study reported 
that the target proportion of steps in the financial intervention group was significantly higher than that in the control group (relative risk=3.71) during the intervention period, ${ }^{7}$ whereas another study reported that the mean proportion of days on which a 7000-step goal was achieved as a result of individual incentive was not significantly higher than in the control group (0.25 vs 0.18$){ }^{8}$

The aim of the present study will be to examine the effect of offering a financial incentive for increasing the number of daily walking steps among physically inactive adults in a community setting.

\section{METHODS}

\section{Study design}

The design is a RCT (single-centre, single-blind, parallel-group study) in which subjects are randomly assigned to one of two groups: an intervention group or a waitlist control group.

\section{Recruitment}

In August 2018, two types of leaflets (preliminary notice and information about recruitment) related to the study will be distributed to each house in the Nakayama area, Aoba-ku, Sendai city, Japan. Posters giving details about recruitment will also be displayed in the Nakayama area. Inclusion criteria and exclusion criteria will be stated on the entry form. Applicants who meet the inclusion criteria and not the exclusion criteria will be able to apply by Web application, FAX or telephone. Considering an estimated attrition of about 10 individuals, we will accept 85 applicants.

\section{Inclusion criteria}

Individuals will be able to apply for participation in this study if they meet all of the following criteria: (1) men and women (aged 20 years or more) living in the Nakayama area, (2) possession of an integrated circuit (IC) Card for Community Development in the Nakayama area (Nakayama Machi-dukuri IC Card), (3) ability to walk unaided without using a cane, Zimmer frame or wheelchair. All the above inclusion criteria will be judged on the basis of self-reports from the participants.

Possession of the IC Card was considered to be an inclusion criterion because it was a means of providing the intervention (financial incentive). The IC Card was developed as a financial incentive to promote physical activity. Persons possessing the IC Card are given shopping points when they go shopping and participate in community activities in the Nakayama area. The IC Card is also intended to enhance social interaction with locals. The intervention in the present study is the first community activity project.

According to the 2015 Population Census, the number of adults (aged 20 years or more) living in Nakayama area was 13734 persons.

\section{Exclusion criteria}

Individuals who meet any of the following criteria will not be able to participate in the study: (1) individuals whose physical activity is restricted by a physician, (2) history of heart attack or stroke within the last 6 months, (3) blood pressure exceeding $180 \mathrm{~mm} \mathrm{Hg}$ systolic or $110 \mathrm{~mm} \mathrm{Hg}$ diastolic, (4) already habitually exercising (task of $\geq 4$ metabolic equivalents) more than two times per week. All exclusion criteria except for blood pressure will be judged on the basis of self-reports from participants.

\section{Study procedure}

Figure 1 illustrates the flow of the study procedure.

In the briefing session in September 2018, the inclusion and exclusion criteria for each applicant will be rechecked by researchers in the study site (the Nakayama Tobinoko House). Chosen subjects will provide informed consent to participate in the study. On the same day, blood pressure measurement, an interview using a questionnaire and explanation about use of a pedometer will then be performed. At the briefing session, each participant will be provided with a pedometer.

The day after the briefing session will be the start date of steps evaluation. We will perform evaluation and feedback about daily steps every 3 weeks in the study site (the Nakayama Tobinoko House). All participants must wear the pedometer every day during the study period (12 weeks).

For all participants, the number of daily steps at the baseline will be measured in first 3 weeks of the study period (table 1). Then, participants who provide their data of daily steps will be randomly assigned to the intervention group or the control group (participants who provide any data ( $\geq 1$ days) at the baseline will be included). At this stage, we assume that approximately 74 persons (ie, the target sample size) would be included in the random assignment.

During the next 3 weeks (intervention period), the participants in the intervention group will be given a chance to gain a financial incentive if they achieve their daily steps goals (for definition of goals, see the next section).

During 7-9 weeks, a chance to gain a financial incentive will not be provided in both the intervention group and the control group. This period (7-9 weeks) is to examine whether the number of steps in the intervention group remain higher than that in the control group even after the incentive period (table 1).

On the other hand, the control group will be given a chance to gain a financial incentive (mentioned above) in the last 3 weeks (9-12 weeks) and thereby all participants will have a fair opportunity to gain such an incentive. The data obtained at 10-12 weeks will not be used for analysis to evaluate the effect.

\section{Intervention}

The intervention is a financial incentive in the form of shopping points, which can be redeemed at facilities in 
Time line

1 weeks

3 weeks

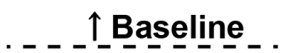

Intervention group

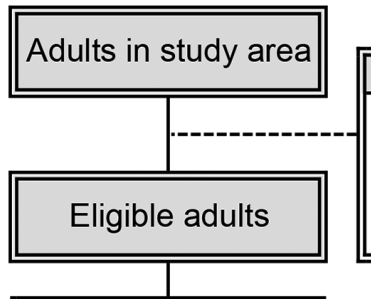

Briefing session

Baseline daily steps (1-3 weeks)

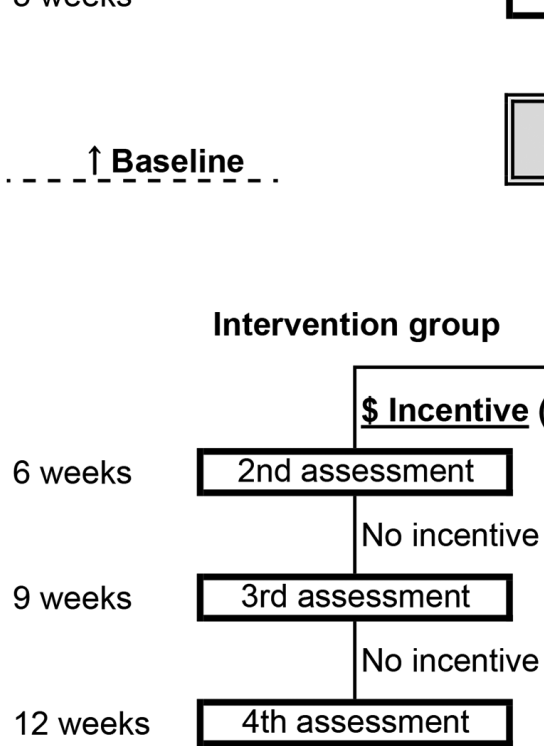

1st assessment

RCT participants

Random assignment (1:1)

2) History of heart attack or sroke.

Severe hypertension.

4) Performing exercise $\geq 2$ times/week

No data available for baseline daily steps

Waitlist control group

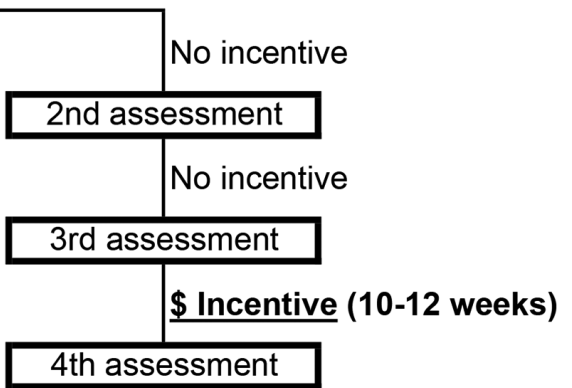

Figure 1 Flowchart of the study procedure.

the study area (14 facilities of Nakayama area). Two kinds of financial incentive will be offered:

1. If the average number of daily steps in the intervention period is $\geq 6000$, shopping points worth 1000 Japanese yen will be awarded.

\begin{tabular}{|c|c|c|}
\hline Time line & $\begin{array}{l}\text { Purpose of } \\
\text { evaluation }\end{array}$ & Hypothesis \\
\hline $1-3$ weeks & $\begin{array}{l}\text { Baseline number } \\
\text { of steps }\end{array}$ & \\
\hline 4-6 weeks & $\begin{array}{l}\text { Effect of } \\
\text { incentive }\end{array}$ & $\begin{array}{l}\text { Is the number of steps } \\
\text { in the intervention } \\
\text { group higher than that } \\
\text { in the control group? }\end{array}$ \\
\hline 7-9 weeks & $\begin{array}{l}\text { Sustained effect } \\
\text { of incentive }\end{array}$ & $\begin{array}{l}\text { Does the number of } \\
\text { steps in the intervention } \\
\text { group remain higher } \\
\text { than that in the control } \\
\text { group even after the } \\
\text { incentive period? }\end{array}$ \\
\hline $10-12$ weeks & $\begin{array}{l}\text { Chance for } \\
\text { waitlist control }^{*}\end{array}$ & \\
\hline
\end{tabular}

*Period for providing a chance of financial incentive for the waitlist control group. Thus, this period will not be included in the statistical analysis of this trial.
2. If the average number of daily steps during the intervention period increases by $\geq 1000$ from the baseline level, shopping points worth 1000 Japanese yen will be awarded.

These daily step targets have already been applied in Japanese national health actions. ${ }^{34}$ National Health Action of Japan has emphasised that an increase of 1000 steps has some impact on population health, because it contributes to a $3.2 \%$ reduction in the average relative risk of non-communicable diseases, dementia, joint-musculoskeletal impairment and mortality. ${ }^{3}$ For example, if a person keeps walking 6500 steps during both the baseline and intervention periods, only financial incentive ' 1 ' (1000 Japanese yen worth of points) will be awarded. If a person walks 3000 steps in the baseline period and 4000 steps in the intervention period, only financial incentive '2' (1000 Japanese yen worth of points) will be awarded. If a person walks 5000 steps in the baseline period and 7000 steps in the intervention period, both financial incentives ' 1 ' and '2' (2000 Japanese yen worth of points) will be awarded. Based on the exchange rate on 31 August 2018, 2000 Japanese yen was equivalent to 14.0 British Pounds.

All participants will be provided shopping points at the same time (after the end of the trial, ie, the 12th week) regardless of the intervention period. 


\section{Waitlist control group}

The waitlist control group is also given a financial incentive in the last 3 weeks (figure 1). All conditions except for timing will be the same as for the intervention group.

\section{Power and sample size}

The sample size was estimated by reference to the average increase in the number of daily steps in a previous study conducted in $2013 .{ }^{7}$ The average difference in the number of daily steps between the intervention group $(\mathrm{n}=24)$ and the control group $(n=16)$ was 1302 (an increase of 2348 steps in the intervention group vs an increase of 1046 steps in the control group) when the intervention group was given an incentive of $\$ 20$ (approximately $¥ 2000$ at the time of the study in 2013). In this previous study, the SD in the control group of the increase was 1711 steps.

Therefore, we assumed the result that an average difference of 1302 steps would be achieved in the intervention period (4-6 weeks) by offering a financial incentive of $¥ 2000$ and setting the SD at 1711 . When an $\alpha$ error of 0.05 and a statistical power of 0.90 was applied, the minimum sample size was 74 persons (37 persons per group). Therefore, a total number of 74 participants (37 participants in each group) was set as the target sample size for analysis. When an $\alpha$ error of 0.05 and statistical power of 0.80 were applied with this sample size (37 participants in each group), an average difference of $\geq 1130$ steps was detectable as statistically significant.

\section{Randomisation}

After confirming their eligibility, enrolled participants will be assigned to one of the two groups (1:1 allocation) based on the permuted block method by computer-generated randomisation. The allocation sequence will be managed by two exclusive researchers of the random assignment.

\section{Blinding}

A blinded endpoint evaluation design will be applied. Only researchers of the random assignment can access the assignment data, and other staffs were blinded to the random assignment. The assignment information was managed in password-locked dedicated storage media. Notification of the assignment by the exclusive researchers of the random assignment will be conducted in a closed room where is separated from the other examination places. In this notification process, the exclusive researchers of the random assignment will warn all participants not to talk about their assignment.

In addition, statistical analyses will be blinded to the assignment. The exclusive researchers of the random assignment will not be involved with the statistical analysis.

\section{Baseline characteristics}

Baseline characteristics will be assessed on the date of the briefing session.

Trained interviewers will conduct an interview to obtain information about medical history, frailty (the Kihon checklist), physical activity, transportation when going out, education level, work, subjective economic status, time affluence (having spare time), pain and falling. We will also measure the blood pressure of each participant.

History of diseases will include stroke, hypertension, myocardial infarction, renal disease, hepatic disease, diabetes mellitus, arthritis, osteoporosis, cancer and dyslipidaemia.

Frailty will be assessed by the Kihon checklist (devised by the Japanese Ministry of Health, Labor and Welfare), which is a 25-item self-administered questionnaire designed to identify frail elderly individuals. ${ }^{9}$ Previous studies have reported the validity of the Kihon checklist. ${ }^{10-13}$

Physical activity will be assessed by the Japan Public Health Centre Physical Activity Questionnaire (JPHC PAQ) ${ }^{1415}$ This questionnaire includes information about the average daily amount of time and frequency spent in work-related (including commuting and housework) physical activity, leisure-time physical activity and sleep. ${ }^{14}$ The total physical activity level is calculated as metabolic equivalents of task-hours per day. The correlation between metabolic equivalents estimated by this questionnaire and daily activities reported in 24 hours records was $0.69 .^{14}$

Transportation when going out will be assessed by asking the question 'What kinds of transportation did you use more than twice per week when you went out in the last 1 month?', for which available responses were: 'walking', 'bicycle', 'motorbike', 'car', 'train', 'bus', 'taxi' or 'other'.

Education level will be assessed by asking the question applied in the 2010 Population Census (Japan) 'Please indicate the last school you graduated from.', for which available responses were: "primary school or junior high school', 'senior high school or middle school (under the old system of education)', 'junior college or higher professional school', 'college, university or graduate school'. ${ }^{16}$

Work will be assessed by asking the question 'Do you do any paid work now?', for which available responses were: ' $\geq 4$ times/week', '2-3 times/week', '1 time/week', '1-3 times/month', 'few times/year' or 'none'.

Subjective household economic status will be assessed by asking the question 'How do you feel about your current household economic situation?', for which available responses will be: 'most affluent', 'more affluent', 'neither more nor less', 'less affluent' or 'non-affluent' (selection from these five choices). ${ }^{17}$

Time affluence (having spare time) will be assessed by asking the question 'Do you have time affluence for rest or leisure in daily life? Alternatively, do not you have time affluence for work, housework, or studies?', for which available responses will be: 'more affluent', 'little affluent', 'less affluent', or 'non-affluent' (selection from these five choices). ${ }^{17}$

Falling will be assessed based on question no. 9 of the Kihon checklist 'Have you experienced a fall in the last year?' .

Pain will be assessed based on the question 'How much pain have you experienced during the last 1 month?', 
for which available responses will be: 'none', 'very mild', 'mild', 'moderate', 'severe' or 'very severe'. ${ }^{18}$ Location of the pain will be also ascertained, for which available responses will be: 'shoulder', 'lower back' or 'knee'.

Blood pressure in a seated position after $3 \mathrm{~min}$ of rest will be assessed using an automated sphygmomanometer HEM-1040 (Omron, Kyoto, Japan). Two measurements taken $3 \mathrm{~min}$ apart will be averaged for analysis.

\section{Outcome measurements}

Daily steps will be counted by a pedometer FS-800 (ESTERA, Saitama, Japan) containing a 3-axis acceleration sensor. Data on daily steps will be automatically recorded in the pedometer for 90 days. On the display of the pedometer, only daily steps in each of the last 14 days (not average steps for the selected period) can be checked. Every 3 weeks, trained staffs will transfer data on the number of steps walked daily recorded by the pedometer to a computer as a Comma-Separated Values file via the Near Field Communication function (not via internet). We will provide a clip-on holder for wearing the pedometer on the waist, and we explain to each participant how to use it. Because the pedometer will record 0 steps if a participant forgets to wear it, we will instruct the participants to wear the pedometer at all times except when sleeping or taking a bath.

Because both effect and adverse effect resulting from falls and pain may be expected as a result of the intervention, we will check any tendencies for incident falls and pain.

Incident falls will be assessed based on question no. 9 of the Kihon checklist. Because the assessment will be conducted every 3 weeks for follow-up, we will modify the timing of this question to 'Have you fallen in the past 3 weeks?'. Incident falls are defined as new episodes of falling after the baseline.

Incident pain will be assessed based on the question 'How much pain have you experienced during the past 3 weeks?', and expressed as a six-point verbal rating scale: 'none', 'very mild', 'mild', 'moderate', 'severe' and 'very severe'. Incident pain is defined as worsening of pain severity after the baseline.

\section{Primary outcome}

The primary outcome is the average increase in the number of daily steps compared with the average number during the baseline period (table 1 , table 2). We will thereby examine whether an increase of more than 1302 steps in 4-6 weeks from the baseline level (mean value for sample size) can be expected, and the increase in the daily number of steps resulting from the financial incentive.

\section{Secondary outcome}

The secondary outcomes will be (1) increase in the number of daily steps by 1000 or more in 4-6 weeks or 7-9 weeks from the baseline level (1-3 weeks); (2) incident falls in 4-6 weeks or 7-9 weeks and (3) incident pain in 4-6 weeks or 7-9 weeks (table 2).

\begin{tabular}{ll} 
Table 2 Study outcomes & \\
\hline Measurement & Definition \\
\hline $\begin{array}{l}\text { Primary outcome } \\
\text { Increase in number of } \\
\text { steps }\end{array}$ & $\begin{array}{l}\text { Mean increase in the average } \\
\text { number of steps (in 4-6 weeks } \\
\text { or 7-9 weeks) compared with the } \\
\text { baseline number. }\end{array}$ \\
$\begin{array}{ll}\text { Secondary outcome } \\
\begin{array}{l}\text { Proportion of } \\
\text { participants who } \\
\text { increase their steps }\end{array}\end{array}$ & $\begin{array}{l}\text { Proportion of participants who } \\
\text { increase their average number of } \\
\text { steps by 1000 from the baseline. }\end{array}$ \\
$\begin{array}{l}\text { Incident falls } \\
\text { Incident rate of falls in 4-6 weeks } \\
\text { or 7-9 } 9 \text { weeks. }\end{array}$ \\
$\begin{array}{l}\text { Incident pain } \\
\text { or 7-9 } 9 \text { weeks. }\end{array}$
\end{tabular}

\section{Statistical analyses}

To compare the primary outcome (average difference), t-test will be applied to examine whether the average daily increases in the number of steps 4-6 weeks and 7-9 weeks from the baseline differ significantly between the intervention group and the control group.

For comparison of secondary outcomes between the intervention group and the waitlist control group at 4-6 weeks and 7-9 weeks, logistic regression models will be applied to examine whether the proportions of participants with an increase of 1000 steps or more are significantly different, and applied to assess the probabilities of incident falls and incident pain, respectively.

In addition, stratified analyses will be conducted to check for any differences in the number of steps in terms of sex, age, frailty, physical activity level, transportation when going out, education level, work, subjective economic status, time affluence (having spare time), pain and obesity.

To apply the intention-to-treat principle, multiple imputations will be conducted to consider the effects of missing values on outcome variables.

All of the above analyses will be performed using SAS V.9.4 (SAS Institute, North Carolina, USA).

\section{ETHICS AND DISSEMINATION Ethical considerations}

The ethics committee of Tohoku University Graduate School of Medicine (Sendai, Japan) has reviewed and approved the study protocol (No. 2018-1-171).

The investigator will explain the research proposal along with the documents and provide enough time for individuals to consider their participation. Handwritten signatures will be required on the consent document. The consent form will guarantee protection of personal information and use of the dataset only for academic purposes. Consent documents will be kept by the principal investigator, and copies will be given to the participants. 
All data on participants will be managed by use of an ID number. Personal information will be strictly managed at Tohoku University Graduate School of Medicine. Personal information will be deleted from the dataset for statistical analysis. After the research period, participant's information data will be disposed of in a prescribed way.

Because this trial is a non-invasive intervention, no Data Monitoring Committee will be organised.

If the research protocol needs to change, the principal investigator must obtain approval from the chief of the research institution through the ethical review committee.

\section{Dissemination of research findings}

Results and findings will be submitted and published in a peer-reviewed scientific journal according to the guidelines of CONSORT for RCTs.

Conflicts of interest among researchers is managed by the Conflict of Interest Management Committee at Tohoku University.

\section{Patient and public involvement}

To improve feasibility about the protocol of this trial in Nakayama area, we discussed with members of Nakayama Community Development Center (non-profit organisation corporation) and members of Nakayama Shopping Street Promotion Association. Additionally, members of Nakayama Neighborhood Association are involved to announce about the recruitment. After the end of the trial, as a collaborative programme with Nakayama Community Development Center and Nakayama Shopping Street Promotion Association, we will hold the debrief session for study report to share results of the trial.

\section{DISCUSSION}

This protocol outlines the objectives of the study and explains the study design.

If this study is conducted in accordance with the present plan, the present study would be first RCT for examining the effect of financial incentives for increasing the number of daily steps in Asian population.

This study has several limitations. First, the intervention involves only one type of financial incentive. Thus, the effect of a change in the corresponding financial incentive or its application (eg, donation) would be unclear. Second, only short-term effects during 9 weeks will be evaluated. Thus, the long-term effect (maintaining a higher number of daily walking steps) of the financial incentive would be unclear. Third, a volunteer bias may exist in the present study. Participants may be more highly motivated to achieve the financial incentive goals in comparison with the total population in the study area. Therefore, the external validity towards non-participants (involuntary participants) will be unclear.

Contributors IT supervised this study and is the guarantor. YT, FT and IT were involved in the design. YT, DN, YL and SZ prepared draft manuscript. SA, KM, SZ, YK,
SM, YL, YS, SB, TY, TO and TS revised the manuscript. YT carried out the statistical calculation. All authors approved submission of this manuscript.

Funding This work was supported by TERUMO Foundation for Life Sciences and Arts.

Competing interests None declared.

Patient consent for publication Not required.

Ethics approval The study protocol was reviewed and approved by the Ethics Committee of Tohoku University Graduate School of Medicine.

Provenance and peer review Not commissioned; externally peer reviewed.

Open access This is an open access article distributed in accordance with the Creative Commons Attribution Non Commercial (CC BY-NC 4.0) license, which permits others to distribute, remix, adapt, build upon this work non-commercially, and license their derivative works on different terms, provided the original work is properly cited, appropriate credit is given, any changes made indicated, and the use is non-commercial. See: http://creativecommons.org/licenses/by-nc/4.0/.

\section{REFERENCES}

1. 2018 Physical Activity Guidelines Advisory Committee. Physical activity guidelines advisory committee scientific report. Washington, DC: U.S. Department of Health and Human Services, 2018.

2. World Health Organization. Global action plan on physical activity 2018-2030: more active people for a healthier world. 2018 http:// www.who.int/iris/handle/10665/272722.

3. Miyachi M, Tripette J, Kawakami R, et al. "+10 min of Physical Activity per Day": Japan is looking for efficient but feasible recommendations for its population. J Nutr Sci Vitaminol 2015;61:S7-9.

4. Nishi N. Monitoring Obesity Trends in Health Japan 21. J Nutr Sci Vitaminol 2015;61:S17-9.

5. The Ministry of Health, Labor and Welfare in Japan. Guideline of financial incentives for health promotion. $2016 \mathrm{https} / /$ www.mhlw.go. jp/stf/houdou/0000124579.html

6. Giles EL, Robalino S, McColl E, et al. The effectiveness of financial incentives for health behaviour change: systematic review and metaanalysis. PLoS One 2014;9:e90347.

7. Harkins KA, Kullgren JT, Bellamy SL, et al. A Trial of Financial and Social Incentives to Increase Older Adults' Walking. Am J Prev Med 2017;52:e123-30.

8. Patel MS, Asch DA, Rosin R, et al. Individual versus team-based financial incentives to increase physical activity: a Randomized, Controlled Trial. J Gen Intern Med 2016;31:746-54.

9. Arai H, Satake S. English translation of the Kihon Checklist. Geriatr Gerontol Int 2015;15:518-9.

10. Satake S, Senda K, Hong YJ, et al. Validity of the Kihon Checklist for assessing frailty status. Geriatr Gerontol Int 2016;16.

11. Sewo Sampaio PY, Sampaio RA, Yamada M, et al. Validation and translation of the Kihon Checklist (frailty index) into Brazilian Portuguese. Geriatr Gerontol Int 2014;14:561-9.

12. Fukutomi E, Okumiya K, Wada T, et al. Relationships between each category of 25-item frailty risk assessment (Kihon Checklist) and newly certified older adults under long-term care insurance: a 24-month follow-up study in a rural community in Japan. Geriatr Gerontol Int 2015;15:864-71.

13. Tomata Y, Hozawa A, Ohmori-Matsuda K, et al. [Validation of the Kihon Checklist for predicting the risk of 1-year incident long-term care insurance certification: the Ohsaki Cohort 2006 Study]. Nihon Koshu Eisei Zasshi 2011;58:3-13.

14. Fujii H, Yamamoto S, Takeda-Imai F, et al. Validity and applicability of a simple questionnaire for the estimation of total and domain-specific physical activity. Diabetol Int 2011;2:47-54

15. Sasai $H$, Nakata $Y$, Murakami $H$, et al. Simultaneous validation of seven physical activity questionnaires used in Japanese cohorts for estimating energy expenditure: a doubly labeled water study. $J$ Epidemiol 2018;28:437-42.

16. Ministry of Internal Affairs and Communications. Population census. 1996 http://www.stat.go.jp/english/data/kokusei/index.html

17. Tomata Y, Tanno K, Zhang S, et al. Subjective household economic status and obesity in toddlers: a cross-sectional study of daycare centers in Japan. J Epidemiol 2019;29:33-7.

18. Kaiho $Y$, Sugawara $Y$, Sugiyama K, et al. Impact of pain on incident risk of disability in elderly Japanese: cause-specific analysis. Anesthesiology 2017;126:688-96. 